\title{
FORT- UND WEITERBILDUNG AUF MODERNISIERUNGSKURS!
}

\section{Liebe Kolleginnen, liebe Kollegen,}

dieses Jahr hatten wir alle gehofft, dass es besser würde. Offensichtlich ist die bisherige Coronapolitik allerdings gescheitert. Da für mich nicht erkennbar ist, dass aus den Fehlern gelernt wird, werden wir die Fort- und Weiterbildungskurse der DAA auch für 2022 wieder verstärkt online durchführen. Auf diese Weise können alle Kollegen sicher ihre Ausbildung erhalten. Die DAA e.V. distanziert sich eindeutig von jeglicher Diskriminierung von Ungeimpften oder Geimpften. Daher freuen wir uns, dass wir ein reichliches Angebot an Fort- und Weiterbildung als Web-Seminar durchführen können. Mein großer Respekt gilt aber auch den vielen Referenten unserer Akademie, die trotz der schwierigen Gesundheitslage bereit sind, Präsenzkurse durchzuführen. Auch wenn Web-Seminare bestimmte Inhalte (Ohrpunkte, Zungenbilder usw.) besser vermitteln können als Präsenzveranstaltungen, so ist doch gerade in der Akupunktur das praktische Arbeiten unter Supervision eines erfahrenen Kollegen dadurch nicht zu ersetzen. Aus diesem Grunde werden wir die Kurse, die einen hohen Praxisanteil haben, weiterhin als Präsenzveranstaltung durchführen. Sollten ungeimpfte Kollegen solcheVeranstaltungen nicht besuchen dürfen, werde ich mit dem Referententeam nach gesetzeskonformen Einzelfalllösungen suchen. Das möchte ich an dieser Stelle versprechen!

TIMMENDORF | Der diesjährige Kongress war Balsam für die Seele. Die anfängliche Unsicherheit, die bei Begegnungen im Foyer zu spüren war, legte sich für einen großen Teil der Teilnehmer am zweiten Tag. 170 Teilnehmer verbrachten 5 wundervolle Kongresstage mit spannenden Vorträgen und Workshops miteinander. Der Studentenkurs war gut besucht und wurde jeden Tag zeitlich überzogen, da aufgrund der großen Begeisterung viele
Fragen gestellt wurden. Die Kollegen Kirchgessner und Kastner haben mit Hingabe den jungen Menschen von der TCM-Ernährung berichtet. Das war auch über den Kongress hinaus ansteckend, wie mir Teilnehmer berichteten. Bereichernd war dieses Jahr auch das neue Programm des Kongressorchesters, das mit viel Applaus bedacht wurde und in der Mediathek nachzuhören ist.

Eine weitere freudige Nachricht: Das Programm des Timmendorf-Kongresses 2022 steht bereits und wird Ihnen zum Jahreswechsel zurVerfügung stehen. Auch einen Studentenkurs wird es wieder geben.

Mediathek | Im Mitgliederbereich finden Sie nunmehr unter E-Campus die Mediathek der DAA e.V. mit vielen interessanten Videos zu verschiedenen Bereichen. Es gibt Beiträge, die jedes Mitglied anschauen kann (z.B. „Basiswissen“ und „Innovation/Technik“) sowie Bereiche, die man nur als Teilnehmer aufrufen kann. Die Mediathek wird in Zukunft ein fester Bestandteil der Methodik der DAA e.V. werden und Ihnen die Fort- und Weiterbildung hoffentlich erleichtern. Wir freuen uns auf Ihre Anregungen und Rückmeldungen.

Abschließend möchte ich noch eine Bitte an Sie richten: ,Treten wir gemeinsam gegen eine Spaltung der Gesellschaft, unserer Familien, Freundes- und Kollegenkreise ein, indem wir aus der Kraft der Mitte leben.“

In diesem Sinne verbleibe ich mit vielen guten Wünschen für den Winter

Ihr

B. Ramme

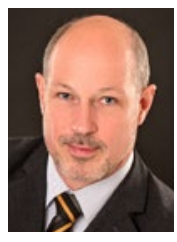

Dr. med. Bernd Ramme

1. Vorsitzender der DAA e.V.

Lerchenfeldstr. 20, D-80538 München

Tel. $+4989 / 8145252$

E-Mail des allg.Büros: kontakt@akupunktur.de, Internet:www.akupunktur.de 